\title{
Portal Cavernoma Cholangiopathy: Consensus Statement of a Working Party of the Indian National Association for Study of the Liver
}

\begin{abstract}
Radha K. Dhiman*, Vivek A. Saraswat ${ }^{\dagger}$, Dominique C. Valla ${ }^{\ddagger}, Y^{*}$ ogesh Chawla ${ }^{\star}$, Arunanshu Behera ${ }^{\S}$, Vibha Varma ${ }^{\|}$, Swastik Agarwal ${ }^{*}$, Ajay Duseja*, Pankaj Puri", Naveen Kalra", Chittapuram S. Rameshbabu**, Vikram Bhatia ${ }^{\star \dagger}$, Malay Sharma ${ }^{\ddagger \ddagger}$, Manoj Kumar ${ }^{\dagger \dagger}$, Subhash Gupta ${ }^{\S \S,\|\|}$, Sunil Taneja ${ }^{\S \S,\|\|}$, Leileshwar Kaman ${ }^{\S}$, Showkat A. Zargar ${ }^{\lceil\pi}$, Samiran Nundy", Shivaram P. Singh"\# , Subrat K. Acharya ${ }^{\star \star \star}$, Jang B. Dilawari ${ }^{\dagger \dagger \dagger}$

"Department of Hepatology, Postgraduate Institute of Medical Education and Research, Chandigarh 160012, India; 'Department of Gastroenterology, Sanjay Gandhi Postgraduate Institute of Medical Sciences, Lucknow, India; ${ }^{\ddagger}$ Service d'Hépatologie, Hôpital Beaujon, Clichy, France; 'Department of Surgery, Postgraduate Institute of Medical Education and Research, Chandigarh 160012, India; "Department of Surgical Gastroenterology and Liver Transplantation, Sir Ganga Ram Hospital, New Delhi, India; "Department of Gastroenterology, Army Hospital (R\&R), New Delhi, India; "Department of Radiodiagnosis, Postgraduate Institute of Medical Education and Research, Chandigarh 160012, India; "Muzaffarnagar Medical College, Muzaffarnagar, India; ${ }^{\dagger \dagger}$ Institute of Liver and Biliary Sciences, New Delhi, India; ${ }^{\ddagger \ddagger}$ Department of Gastroenterology, Jaswant Rai Speciality Hospital, Meerut, India; ${ }^{\S}$ Department of Surgical Gastroenterology \& Liver Transplantation, Indraprastha Apollo Hospital, New Delhi, India; "IIDepartment of Gastroenterology and Hepatology, Indraprastha Apollo Hospital, New Delhi, India; "Department of Gastroenterology, Sher-i-Kashmir Institute of Medical Sciences, Srinagar, India; \#\#Department of Gastroenterology, S.C.B. Medical College, Cuttack, India; ${ }^{\star * *}$ Department of Gastroenterology, All India Institute of Medical Sciences, New Delhi, India; and

${ }^{\dagger \dagger}$ Department of Gastroenterology, Inver Clyde Royal Hospital, Greenock, UK
\end{abstract}

\begin{abstract}
Portal cavernoma cholangiopathy (PCC) is defined as abnormalities in the extrahepatic biliary system including the cystic duct and gallbladder with or without abnormalities in the 1st and 2nd generation biliary ducts in a patient with portal cavernoma. Presence of a portal cavernoma, typical cholangiographic changes on endoscopic or magnetic resonance cholangiography and the absence of other causes of these biliary changes like bile duct injury, primary sclerosing cholangitis, cholangiocarcinoma etc are mandatory to arrive a diagnosis. Compression by porto-portal collateral veins involving the paracholedochal and epicholedochal venous plexuses and cholecystic veins and ischemic insult due to deficient portal blood supply or prolonged compression by collaterals bring about biliary changes. While the former are reversible after porto-systemic shunt surgery, the latter are not. Majority of the patients with PCC are asymptomatic and approximately $21 \%$ are symptomatic. Symptoms in PCC could be in the form of long standing jaundice due to chronic cholestasis, or biliary pain with or without cholangitis due to biliary stones. Endoscopic retrograde cholangiography has no diagnostic role because it is invasive and is associated with risk of complications, hence it is reserved for therapeutic procedures. Magnetic resonance cholangiography and portovenography is a noninvasive and comprehensive imaging technique, and is the modality of choice for mapping of the biliary and vascular abnormalities in these patients. PCC is a progressive condition and symptoms develop late in the course of portal hypertension only in patients with severe or advanced changes of cholangiopathy. Asymptomatic patients with PCC do not require any treatment. Treatment of symptomatic PCC can be approached in a phased manner, coping first with biliary clearance by nasobiliary or biliary stent placement for acute cholangitis and endoscopic biliary sphincterotomy for biliary stone removal; second, with portal decompression by creating portosystemic shunt; and third, with persistent biliary obstruction by performing second-stage biliary drainage surgery such as hepaticojejunostomy or choledochoduodenostomy. Patients with symptomatic PCC have good prognosis after successful endoscopic biliary drainage and after successful shunt surgery. (J CLIN ExP НеPATOL 2014;4:S2-S14)
\end{abstract}

\footnotetext{
Keywords: cholestasis, extrahepatic portal venous obstruction, gallbladder varices, obstructive jaundice, portal hypertensive biliopathy Received: 1.2.2014; Accepted: 2.2.2014

Address for correspondence: Radha K. Dhiman, Professor, Department of Hepatology, Postgraduate Institute of Medical Education and Research, Chandigarh 160012, India. Tel.: +91 172 2756337; fax: +91 1722744401

E-mail: rkpsdhiman@hotmail.com

Abbreviations: CBD: common bile duct; CHD: common hepatic duct; CT: computed tomography; ERC: endoscopic retrograde cholangiography; EUS: endoscopic ultrasound; EHPVO: extrahepatic portal venous obstruction; GRADE: Grading of Recommendations, Assessment, Development and Evaluation; INASL: Indian National Association for Study of the Liver; MRC: magnetic resonance cholangiography; MRI: magnetic resonance imaging; NCPF: non-cirrhotic portal fibrosis; PVT: portal vein thrombosis; PSS: portosystemic shunt; USG: ultrasound; UDCA: ursodeoxycholic acid http://dx.doi.org/10.1016/j.jceh.2014.02.003
} 
B iliary changes seen in association with cavernomatous transformation of the portal vein have been described by various authors since the first report of symptomatic biliary obstruction due to collateral vessels in a patient with extrahepatic portal venous obstruction (EHPVO) in 1944 by Fraser and Brown. ${ }^{1}$ Subsequently biliary changes were also described in relation to other causes of portal hypertension like cirrhosis and non-cirrhotic portal fibrosis (NCPF) albeit with a lower frequency than seen in EHPVO. $^{2-4}$ Authors have used differing nomenclatures and variable criteria to define these biliary changes. ${ }^{5}$ Due to the lack of a standard definition and uniform inclusion criteria in these studies, the clinical significance, natural history and prognosis of these biliary changes remain poorly defined and the management is not standardized. The Indian National Association for Study of the Liver (INASL) set up a working party in 2012 to fill these lacunae and guide clinicians by proposing a standard definition, uniform inclusion criteria for further research and a rational management algorithm. The final report of the INASL Working Party was presented at the annual meeting of the INASL on 23rd March 2013. This is the first-ever Consensus Statement developed on this subject.

\section{THE FOLLOWING QUESTIONS WERE ADDRESSED BY THE WORKING PARTY}

\section{Definition and Nomenclature}

What is the most appropriate definition for the spectrum of biliary changes seen in association with portal hypertension? Of the many biliary changes described on cholangiography, which ones have been consistently reproduced in the literature and are suitably sensitive and specific for this condition? Is the mere presence of portal hypertension of any etiology enough to produce these changes, or is the presence of a portal cavernoma mandatory? Should portal hypertension due to cirrhosis and NCPF be considered as an etiology? What would be most appropriate nomenclature based on the results of the above answers?

\section{Pathogenesis}

What is the relative contribution of mechanical compression by collaterals and ischemia in the development of biliary changes?

\section{Prevalence and Clinical Features}

What is the prevalence of the set of biliary changes seen in portal hypertension? How many of these patients are symptomatic? What are the common presentations? How are patients with symptomatic disease different from asymptomatic disease?

\section{Diagnosis}

Among the large number of changes seen in the biliary tree among patients with portal hypertension, which are characteristic diagnostic features on imaging? Which imaging modality is best for the screening and confirmation of these changes?

\section{Natural History and Prognosis}

hat is the natural course of progression of biliary changes clinically and radiologically? How do these patients fare on follow-up?

\section{Treatment}

What are the relative roles of endoscopic and surgical management in this condition?

\section{QUALITY OF EVIDENCE ON WHICH A RECOMMENDATION IS BASED}

The Working Party adopted the Grading of Recommendations, Assessment, Development and Evaluation (GRADE) system for evaluating evidence. The group assessed existing evidence and, based on its strength, ranked its recommendations as strong (1) or weak (2) and rated level of evidence as high $(\mathrm{A})$, moderate $(\mathrm{B})$ or low $(\mathrm{C}){ }^{6}$

\section{HISTORICAL PERSPECTIVE, NOMENCLATURE AND DEFINITION}

Fraser and Brown first reported symptomatic biliary obstruction due to collateral vessels in a patient with EHPVO in 1944. ${ }^{1}$ Only sporadic reports appeared over the next few decades ${ }^{7-9}$ illustrating the rarity of this condition in the West. Though surgical hazards posed by common bile duct (CBD) varices were recognized in the late 1970 s, ${ }^{10}$ it was the seminal paper by Webb and Sher$\operatorname{lock}^{11}$ that focussed attention on this condition. Choledochal varices were first demonstrated on cholangiography by Williams in $1982,{ }^{12}$ which was later confirmed by other workers. $^{13,14}$ That collateral decompression by portosystemic shunt (PSS) surgery can lead to reversal of biliary obstruction was first reported in 1988, ${ }^{15}$ an observation corroborated and qualified subsequently. ${ }^{16,17}$ The era of endoscopic management in this condition began in 1993 with the first report of endoscopic biliary stenting in this condition. ${ }^{18}$ Definition of biliary changes seen in portal hypertension has varied widely between studies. Sarin et $\mathrm{al}^{19}$ have defined portal biliopathy broadly as biliary ductal and gallbladder wall abnormalities seen in patients with portal hypertension. While this definition is simple it is not specific and includes biliary changes due to any other coincidental disease in a patient who happens to have portal hypertension also. ${ }^{19}$ The definition proposed 
by Dhiman et $\mathrm{al}^{20}$ was more descriptive defining portal hypertensive biliopathy as abnormalities of the entire biliary tract including intrahepatic and extrahepatic bile ducts, cystic duct and gallbladder in patients with portal hypertension. However, even this definition failed to specify which biliary changes are to be included and in which portions of the biliary tree the changes are mandatory. ${ }^{20}$ A definition which would be specific to this condition so as to have reproducibility between studies from different authors on this subject was missing. Hence the working party debated the definition on two fronts: 1 . Which of the many biliary changes described on cholangiography have been consistently reproduced in the literature and are sensitive and specific for this entity? 2. Should portal hypertension of any etiology be considered in the definition or is the presence of a cavernoma mandatory?

Most of the studies had reported that the extrahepatic biliary ducts were almost invariably involved, while some also described changes in the gallbladder and cystic duct. Changes had also been described up to the 2 nd generation bile ducts. While similar biliary changes have been reported in other causes of portal hypertension, in a majority of the studies typical changes had been described in patients with EHPVO and portal cavernoma. Biliary changes described in patients with cirrhosis and NCPF are primarily intrahepatic and likely be due to parenchymal disease; absence of portal cavernoma also explains the absence of biliary changes in extrahepatic ducts in patients with cirrhosis and NCPF. Since the natural history, prognosis and response of the biliopathy to therapy is expected to vary based on the underlying cause of portal hypertension, the presence of a portal cavernoma was considered to be a part of the definition to maintain uniformity between studies and to ensure a homogenous patient population.

The nomenclature of biliary changes secondary to portal hypertension has been even more varied than the definitions. Dilawari and Chawla ${ }^{21}$ described it as "pseudosclerosing cholangitis" on the basis of findings similar to sclerosing cholangitis but in the absence of cholangitis or features of primary sclerosing cholangitis in the majority of patients. Bayraktar et al described it as the "pseudocholangiocarcinoma sign" due to endoscopic retrograde cholangiography (ERC) similarities with cholangiocarcinoma. ${ }^{22}$ Sarinet $\mathrm{al}^{19}$ named it "portal biliopathy" to reflect the biliary changes secondary to portal hypertension. Similarly Malkan et al. ${ }^{20}$ called it "cholangiopathy associated with portal hypertension". Subsequently the entity was called "portal cavernoma associated cholangiopathy" as almost all patients had portal cavernoma at surgery. ${ }^{23}$ Dhiman et al named it "portal hypertensive biliopathy" as the entire or part of the biliary tract is involved in these patients, and these abnormalities could be due to portal hypertension of any etiology, and not necessarily portal cavernoma. ${ }^{21},{ }^{24}$ Other terms that that have been used include "extrahepatic portal biliopathy"25 "vascular biliopathy", ${ }^{26}$ "ischemic cholangiopathy", 27 "portal ductopathy", ${ }^{28}$ and "portal cholangiopathy" ${ }^{29}$

The term "Portal cavernoma cholangiopathy (PCC)" was agreed upon as the consensus nomenclature by the Working Party since it implied the presence of a portal cavernoma resulting in abnormalities of the biliary tree including extra- and intra-hepatic bile ductular system, gallbladder and cystic duct.

The consensus nomenclature and definition arrived at by the Working Party was as follows:

\section{Consensus Statement}

1. Portal cavernoma cholangiopathy is defined as abnormalities in the extrabepatic biliary system including the cystic duct and gallbladder with or without abnormalities in the 1 st and 2nd generation biliary ducts in a patient with portal cavernoma. For the diagnosis to be established all of the following criteria would have to be fulfilled: 1 presence of a portal cavernoma, 2 typical cholangiographic changes on ERC or magnetic resonance cholangiography (MRC) (Table 1) and 3 absence of other causes of these biliary changes like bile duct injury, primary sclerosing cholangitis, cholangiocarcinoma etc (Table 2). (Strength-1, Evidence-A)

\section{VASCULAR ANATOMY OF THE COMMON BILE DUCT}

It has conventionally been believed that the intrahepatic and extrahepatic bile ducts are totally dependent on the hepatic arterial supply for oxygenation. However, recently Slieker et $\mathrm{al}^{30}$ demonstrated that microvascular blood flow through the CBD decreased to $62 \%$ of normal on clamping the portal vein in 15 patients undergoing pancreaticoduodenectomy suggesting the importance of portal vein in biliary vascular supply. About $50 \%$ of hepatic arterial blood is meant for the supply of biliary tract. The supraduodenal bile duct, which includes the common hepatic duct (CHD) and $\mathrm{CBD}$ on imaging, has the poorest vascularization and is most vulnerable to ischemic changes. ${ }^{27}$ The retropancreatic, hilar and intrahepatic portions of the bile ducts have

Table 1 Cholangiographic Abnormalities of Portal Cavernoma Cholangiopathy.

\begin{tabular}{l}
\hline Extrinsic impressions/indentations \\
Shallow impressions/indentations \\
Irregular ductal contour \\
Stricture \\
Filling defects \\
Bile duct angulation \\
Upstream dilatation \\
Ectasia \\
\hline
\end{tabular}


Table 2 Differential Diagnosis of Portal Cavernoma Cholangiopathy.

\begin{tabular}{l}
\hline Primary sclerosing cholangitis \\
\hline Bile duct neoplasms \\
\hline Biliary tract surgery \\
Recurrent pyogenic cholangitis \\
Acquired immunodeficiency syndrome cholangiopathy \\
\hline Biliary parasitosis \\
Choledocholithiasis \\
Congenital abnormalities of the biliary tract \\
Ischemic bile duct stricture \\
Toxic bile duct strictures \\
Strictures due to autoimmune and chronic pancreatitis \\
\hline
\end{tabular}

relatively copious arterial blood supply. ${ }^{31}$ Starting from the ampulla of Vater, the entire biliary system derives arterial blood supply from the branches of the celiac trunk. ${ }^{32}$ The arrangement of arterial and venous plexuses of CBD is similar. Epicholedochal plexus on the surface of the CBD is formed from the branches of the paracholedochal vessels. The paracholedochal and epicholedochal plexuses connect with the intramural and subepithelial plexuses in the wall of the CBD via perforator vessels that pierce its wall. ${ }^{33}$

The venous drainage of the CBD is arranged in the form of two plexuses. The epicholedochal venous plexus of Saint is a fine reticular plexus on the surface of the bile ducts. The paracholedochal venous plexus of Petren runs outside and parallel to the bile ducts. Two marginal veins known as 3 o'clock and 9 o'clock marginal veins are formed by the paracholedochal plexus, with, occasionally, an additional 6 o'clock marginal vein seen on posterior surface of CBD. Inferiorly these veins communicate with gastric veins, posterior superior pancreaticoduodenal vein, and gastrocolic trunk while superiorly the marginal veins join the hilar venous plexus to drain into adjacent portal vein branches or enter into the hepatic substance. Detailed arterial supply and venous drainage of the biliary system has been described in an article in this issue of the Journal. $^{34}$

In EHPVO multiple porto-portal collateral veins with hepatopetal flow develop to bypass the obstructed segment of the portal vein. These collaterals involve the paracholedochal and epicholedochal venous plexuses and cholecystic veins. The paracholedochal veins dilate first causing external compression of the CBD wall with protrusion of dilated paracholedochal veins into the thin and pliable wall of the CBD. The normally smooth intraluminal surface of the CBD becomes irregular due to the dilated epicholedochal venous collaterals. The pressure is transmitted via the perforators to the sub-epithelial plexus resulting in the development of subepithelial varices in the CBD wall which can cause bleeding. ${ }^{35}$ Cavernoma formation has been demonstrated as early as within 6-20 days of acute portal vein thrombosis (PVT) with intrahepatic extension of the cavernoma seen in $76 \%$ of patients. ${ }^{36}$ Both porto-systemic (chiefly through left gastric vein) and porto-portal (periportal and pericholecystic venous channels) collaterals are seen, with all the vessels showing hepatopetal blood flow. ${ }^{36}$

\section{Consensus Statement}

2. General pattern of arterial supply and venous drainage of the bile ducts is quite similar. Fine branches from the posterior superior pancreaticoduodenal, retroportal, gastroduodenal, hepatic and cystic arteries form two plexuses to supply the bile ducts. The paracholedochal plexus, as right and left marginal arteries, runs along the margins of the bile duct and the reticular epicholedochal plexus lies on the surface. The retropancreatic, hilar and intrabepatic parts of biliary tract have copious supply, but the supraduodenal bile duct has the poorest vascularization and hence is susceptible to ischemic changes. (Strength-1, Evidence-A)

3. Two venous plexuses drain the biliary tract. The fine reticular epicholedochal venous plexus of Saint on the wall of the bile duct drains into the paracholedochal venous plexus of Petren (also called marginal veins or parabiliary venous system) which in turn is connected to the posterior superior pancreaticoduodenal vein, gastrocolic trunk, right gastric vein, superior mesenteric vein inferiorly and intrabepatic portal vein branches superiorly. (Strength-1, Evidence-A)

4. In conditions of porto-mesenteric venous obstruction, these plexuses enlarge to form a portal cavernoma surrounding the bile duct and bring about morphological changes observed in portal cavernoma cholangiopathy. (Strengtb-1, Evidence-B)

\section{PATHOGENESIS}

Acute portal vein occlusion causes loss of portal blood flow, which is initially compensated by the hepatic artery buffer response, resulting in dilatation of the hepatic arterial system. $^{37,38}$ Subsequently, rapid development of venous collaterals and formation of varices as well as cavernoma occur. These dilated venous collaterals exert external pressure and protrude into the thin and pliable CBD and the hepatic ducts (Figure 1). ${ }^{17,23,24,39}$ The dilatation of the plexus of Saint causes fine irregularities in the biliary tract while the dilatation of the plexus of Petren causes extrinsic compression over bile duct. ${ }^{17}$ The frequent involvement of the left hepatic duct in PCC could be due to prominent collateral veins at the junction of the umbilical vein and left branch of the portal vein. ${ }^{40}$ Intra-ductal 

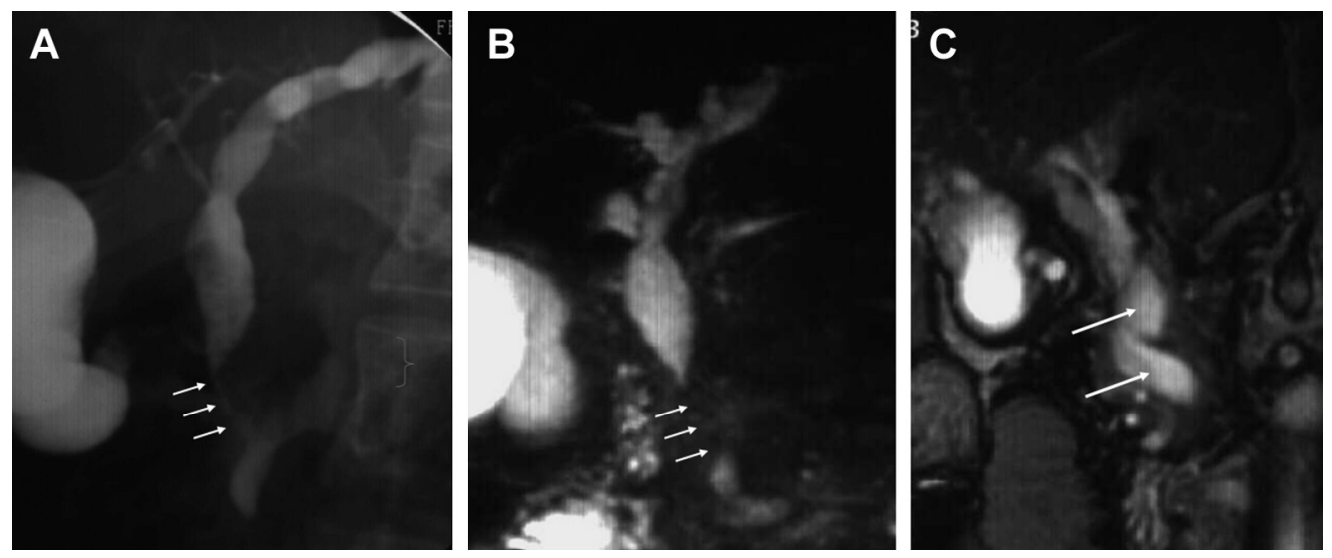

Figure 1 (A) Endoscopic cholangiography shows common bile duct stricture (arrows). (B) MR cholangiography confirms common bile duct stricture seen on endoscopic cholangiography (arrows). (C) MR venography shows that common bile duct stenosis is caused by pressure from a large collateral. Blood vessels appear in white in flow sensitive sequence (large arrows).

collaterals may also contribute to the biliary abnormalities as evidenced by endoscopic ultrasound (EUS) demonstration of paracholedochal varices which perforate the wall of the bile duct to lie in the subepithelial layer, causing biliary irregularity. ${ }^{35,41}$ Evidence in favor of compression as a cause of biliary changes comes from imaging evidence of collaterals impinging on the bile ducts at the site of biliary irregularities ${ }^{17,23}$ and from the reversal of biliary changes after shunt surgery in some patients. ${ }^{42}$

However, not all biliary changes reverse after shunt surgery. This could be due to incomplete disappearance of varices after surgery, presence of a fibrous tumor-likecavernoma causing persistent compression ${ }^{17,23,43}$ or irreversible irregularities due to ischemic changes within the bile duct secondary to deficient portal blood supply or prolonged compression by collaterals. Also, not all biliary strictures correlate with collaterals on imaging with MRC coupled with MR venography. In a study by Dhiman et al ${ }^{17}$ only $55 \%$ of dominant biliary strictures were caused by compression from adjacent collaterals whereas no such relationship was seen in the remaining $45 \%$, which were presumed to have an ischemic origin. Decrease in biliary blood flow on portal vein clamping has been described in humans by Seiker et $\mathrm{al}^{30}$ Portal branch ligation in rabbits results in slight dilatation of the bile ducts of affected lobes. ${ }^{44}$ It is tempting to speculate that development of biliary changes in PCC is due to two components a)a reversible component, due to biliary compression by engorged collaterals, that reverses after shunt surgery and b) a fixed component that does not reverse after shunt surgery and is likely due to ischemic changes in the bile duct, occurring either at the time of portal vein thrombosis or due to prolonged compression by collaterals or by a solid, tumor-like 'fibrotic' cavernoma, containing connective tissue. Detailed pathogenesis of PCC has been described in an article in this issue of the Journal. ${ }^{42}$

\section{Consensus Statement}

5. Biliary changes seen in PCC may be due to compression by large collaterals or may be ischemic, due to portal vein thrombosis and/or prolonged biliary compression. While the former are reversible after porto-systemic shunt surgery, the latter are not. Collateral compression and ischemic injury, are not mutually exclusive in a patient with PCC. (Strength-1, Evidence-A)

\section{PREVALENCE AND CLINICAL CHARACTERISTICS}

The prevalence of biliary changes on cholangiography ranges from 78 to $100 \%$ in most series of patients with EHPVO. However, symptoms are present in only $5-50 \%$ of patients (Table 3). ${ }^{45}$ Symptoms could be in the form of long standing jaundice due to chronic cholestasis, or biliary pain with or without cholangitis due to biliary stones. ${ }^{20}$ Jaundice is invariably present in patients with symptomatic PCC, and was present at diagnosis in about $2 / 3$ rd of patients, while it was the sole manifestation of EHPVO (without preceding variceal bleeding) in $15 \%$ of patients managed surgically. ${ }^{46}$ Jaundice is usually mild; mean serum bilirubin level was $2.7 \mathrm{mg} / \mathrm{dL}$ (range $0.7-16$ ) in one study while only $15 \%$ of patients had a bilirubin value of more than $5 \mathrm{mg} / \mathrm{dL}$ in another study. ${ }^{20,47}$ Half to $2 / 3$ rd of patients have experienced cholangitis, with number of episodes ranging from 1 to 25 . Abdominal pain is seen in about half of symptomatic PCC whereas awareness of splenic lump is reported in only a minority of cases. ${ }^{4-48}$ Diagnosis of EHPVO antedates symptomatic PCC by $8-10$ years. Age of the patient and duration of EHPVO are chief risk factors for development of symptoms in PCC. Patients with symptomatic PCC were on average 14 years older at ( 35 years versus 21 years age) and had been diagnosed with EHPVO for 7 years longer (11 years versus 4 years) compared to asymptomatic PCC. ${ }^{20}$ 
Table 3 Frequency of Biliary Changes in Patients with Extra-hepatic Portal Venous Obstruction.

\begin{tabular}{|c|c|c|c|c|c|c|}
\hline Study & Year & $\mathbf{n}$ & $\begin{array}{l}\text { Age mean } \\
\text { and/or range }\end{array}$ & $\mathbf{M} / \mathbf{F}$ & $\begin{array}{l}\text { Frequency of biliary } \\
\text { changes (\%) }\end{array}$ & $\begin{array}{c}\text { Symptomatic } \\
\text { patients (\%) }\end{array}$ \\
\hline Dilawari and Chawla ${ }^{21}$ & 1992 & 20 & $22(13-38)$ & $16 / 4$ & 100 & 5 \\
\hline Sarin et $\mathrm{al}^{19}$ & 1992 & 20 & $9-32$ & $16 / 4$ & 90 & 15 \\
\hline Khuroo et $\mathrm{al}^{49}$ & 1993 & 21 & $14 \pm 8.8$ & $13 / 8$ & 81 & 38 \\
\hline Bayraktar et al $^{22}$ & 1995 & 44 & $31.5(9-67)$ & $24 / 20$ & 94 & 30 \\
\hline Malkan et $\mathrm{al}^{2}$ & 1999 & 20 & 23 & $12 / 8$ & 85 & 10 \\
\hline Nagi et al ${ }^{50}$ & 2000 & 43 & $14-45$ & $25 / 18$ & 100 & 19 \\
\hline Condat et $\mathrm{al}^{23}$ & 2003 & 25 & 49.5 & $15 / 1$ & 84 & 28 \\
\hline Sezgin et al ${ }^{51}$ & 2003 & 36 & NA & NA & 94 & 10 \\
\hline Dhiman et $\mathrm{al}^{24}$ & 2006 & 53 & $24.5(13-56)$ & $36 / 17$ & 100 & 24.5 \\
\hline 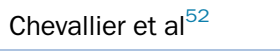 & 2006 & 10 & $43.5(29-56)$ & $5 / 5$ & 90 & 50 \\
\hline Llop et $\mathrm{al}^{29}$ & 2011 & 67 & $47(19-77)$ & $41 / 26$ & 78 & 21 \\
\hline Total [median (range)] & & 262 & & & $90(78-100)$ & $21(5-50)$ \\
\hline
\end{tabular}

NA, not available.

Gallstones and CBD calculi are other major risk factors for the development of symptoms. In one study $95 \%$ patients with symptomatic PCC had dilated intrahepatic biliary radicles and $82 \%$ had dilated CBD on ultrasound. Gallstones were seen in $1 / 3$ rd and bile duct stones in $18 \%$ of symptomatic patients. ${ }^{46}$ In another study, gallstones and CBD stones were seen in $54 \%$ and $23 \%$ of symptomatic PCC patients respectively compared to $0 \%$ and $2.5 \%$ respectively in asymptomatic PCC patients. ${ }^{20}$ Prevalence and clinical characteristics of PCC have been described in an article in this issue of the Journal. ${ }^{45}$

\section{Consensus Statement}

6. Majority of the patients with PCC are asymptomatic. (Strength-1, Evidence-A)

7. Approximately $21 \%$ (range 5-50\%) of patients with PCC are symptomatic. (Strength-1, Evidence-A)

8. Risk factors for development of symptomatic PCC include older age of the patient, long duration of EHPVO, dilated segments of bile ducts, and the presence of gallstones and CBD stones (Strength-2, Evidence-C).

\section{DIAGNOSIS}

\section{Endoscopic Retrograde Cholangiography}

ERC has traditionally been the gold standard for diagnosis of PCC. However due to its invasive nature, risk of complications and need for sequential imaging it is now being replaced by non-invasive radiological modalities for diagnosis with ERC being reserved for therapy. Standardized nomenclature (Table 1) and definitions of various findings on ERC findings in PCC, are proposed by the Working Party in this issue of the Journal. Based on currently available literature reports rather than on rigorously validated data, this terminology is expected to provide a framework for ensuring uniformity in future studies. Nomenclature and definitions of various findings on ERC findings in PCC have been described in an article in this issue of the Journal. ${ }^{53}$ Briefly, nomenclature and definitions of ERC findings are as follows:

i) Extrinsic impressions/indentations: smooth thumb-like impressions on the bile duct, with a nodular contour. The indentation is more than one-quarter of the width of the opacified duct. Impressions may be multiple.

ii) Shallow impressions/indentation(s): smooth noncontiguous impressions on the bile duct, less than one-quarter of ductal diameter.

iii) Irregular ductal contour: fine-wavy, irregular contour of the bile duct walls due to contiguous shallow indentations, less than one-quarter of the ductal diameter.

iv) Stenosis: variable length narrowing of the ductal lumen, in reference to well opacified downstream duct segment. Stenoses might be associated with upstream dilatation and may be due to extrinsic compression by collaterals or intrinsic narrowing or stricturing due to mural fibrosis. Strictured bile duct segments should offer some resistance to passage of an adequately inflated extraction balloon across it and should produce a waist on non-compliant balloons inflated within the narrowed segment. Stenoses and strictures may be divided into 'mild to moderate' or'severe' depending on whether the narrowed segment is > or < two-third of the diameter of adjacent normal segment.

v) Upstream dilatation: proximal dilatation can be similarly classified as 'mild to moderate' or 'severe', depending on whether the dilated segment is between $1.5-2$ or $>2$ diameter of the adjacent normal duct, respectively.

vi) Filling defects: round, oval, or elongated defects in the cholangiographic image, with contrast on three or all 
sides. Filling defects can represent stones, prolapsing intra-luminal varices, or clots.

vii) Bile duct angulation: it is proposed that an angle of $\leq 145^{\circ}$ between lower and upper CBD be considered as significant

viii) Ectasia: dilated segment of biliary tree without any evident downstream obstruction

Cholangiographic findings in PCC were first classified by Chandra et $\mathrm{al}^{4}$ who proposed a system based on ERC which has been applied to MRC as well, based on the distribution of lesions. Type I is involvement of extrahepatic ducts only, type II is involvement of intrahepatic bile ducts only, type IIIa is extrahepatic bile duct with unilateral intrahepatic bile duct involvement and type IIIb is extrahepatic bile duct with bilateral intrahepatic bile duct involvement.

Llop et $\mathrm{al}^{29}$ have graded the severity of abnormalities of the biliary treein PCC according to cholangiographic findings into grade I: irregularities or angulations of the biliary tree, grade II: indentations or strictures without upstream biliary dilation and grade III: strictures with upstream biliary dilation. Dilatation is defined as ductal diameter of $>7 \mathrm{~mm}$ for extra-hepatic duct and/or $>4 \mathrm{~mm}$ for intra-hepatic ducts.

The INASL Working Party suggests that, for future studies in PCC, standardized nomenclature and terminology proposed-above should be used for describing lesions while the system proposed by Llop et al should be used for grading severity of cholangiographic changes, since not only is it based on the severity of cholangiographic findings but has also been prospectively validated in contrast to the classification proposed by Chandra et $\mathrm{al}^{4}$ which deals only with the distribution of changes. However, it is acknowledged that classification and grading systems are likely to change as more severe grades of abnormalities, more extensive distribution of changes and multifocal changes are reported in PCC.

\section{Ultrasonography}

Combination of ultrasound (USG) with color Doppler is the initial screening modality for the PCC as it is non-invasive, free of radiation, easily available and relatively cheap. Findings on sonography vary from non-visualization of portal vein to a completely thrombosed vein with cavernoma formation seen as multiple tubular anechoic structures in the porta. Color Doppler sonography demonstrates flow in portal collaterals in the absence of flow in portal vein. 30-50\% cases may have tortuous collaterals around the wall of the gallbladder and rarely color Doppler may pick up varices in the wall of the gallbladder and the bile duct as thickened wall with color flow on Doppler imaging. ${ }^{54}$ USG can detect narrowing or stenosis of the CBD with associated proximal dilatation and associated cholelithiasis and/or choledocholithiasis. However the CBD can get obscured by high level echoes in the porta hepatis and by the multiple collateral channels. Exact details of biliary narrowing and differentiation between extrinsic compression and ischemic stricturing are not possible on USG. Signs of cirrhosis, associated portosystemic collaterals and splenomegaly are well detected with USG and color Doppler.

\section{Computed Tomography (CT) or Magnetic Resonance Imaging (MRI)}

Cross-sectional imaging with contrast enhanced computed tomography (CT) or magnetic resonance imaging (MRI) confirms features of PCC but is mainly needed to exclude other possible causes of the observed changes such as biliary malignancy and also to search for possible cause of portal vein obstruction such as chronic pancreatitis. MRI is preferred to CT as it does not entail radiation exposure and is superior to $\mathrm{CT}$ in detailing biliary anatomy. Combination of contrast enhanced MRI or MR portovenography with MRC have currently replaced ERC as the diagnostic imaging of choice for PCC. ${ }^{23,40,55}$ In addition to detailed delineation of the biliary abnormalities including differentiation of stones from intramural varices, they delineate the entire spleno-portal axis, help in planning PSS surgery, and depict the relationship of collaterals with the biliary tract which is not possible with ERC (Figure 1). MRI also differentiates epicholedochal collaterals, appearing as dot like enhancing structures in the bile duct wall, from paracholedochal collaterals and gallbladder varices which appear as low signal intensity channels on $\mathrm{T} 2$-weighted images and as enhancing tortuous collaterals on dynamic 3D gradientecho images. ${ }^{56}$ Typical biliary findings of PCC seen on MRC are similar to those seen on ERC, ${ }^{40}$ are listed in Table 1 and are reviewed in detail elsewhere in this issue. ${ }^{57}$ Recent MRC studies of PCC have reported that type I and type III abnormalities according to Chandra et al, ${ }^{4}$ i.e. involvement of extrahepatic ducts alone or in combination with the intrahepatic ducts, were the most frequent findings, extrahepatic bile ducts being involved almost universally involved. ${ }^{56}$ No discrepancy between MRC and ERC in the biliary findings of PCC in patients appeared when both investigations were performed. ${ }^{40,55,56}$ Hence MRCP with MR portovenography is the modality of choice for mapping of the biliary and vascular abnormalities.

\section{Endoscopic Ultrasound}

Role of EUS in the diagnosis and management of PCC is evolving. There is no study directly comparing ERC or MRC with EUS. On EUS, biliary varices appear as multiple, large, serpiginous, anechoic vascular channels in and/or around the extrahepatic biliary tract. ${ }^{58}$ EUS with Doppler can accurately differentiate paracholedochal, epicholedochal, intracholedochal and subepithelial varices. This distinction is especially relevant if a subsequent ERC is 
planned, since the presence of intracholedochal and subepithelial varices may increase risk of bleeding during stone extraction and stricture dilatation respectively. ${ }^{59,60} \mathrm{~A}$ detailed comparison of various imaging modalities useful for the diagnosis of PCC has been reviewed elsewhere in this issue. ${ }^{60}$

\section{Consensus Statement}

9. ERC has no diagnostic role because it is invasive and is associated with risk of complications. ERC is reserved for therapeutic procedures. (Strength-1, Evidence-A)

10. A standardized nomenclature and definition of various findings on ERC has been proposed for uniformity in future studies and need prospective validation in future studies (Strength-1, Evidence-B).

11. USG with Color Doppler should be the initial imaging modality for suspected cases of PCC (Strength-1, Evidence-B).

12. MRC with MR portovenography is a noninvasive and comprehensive imaging technique, and is the modality of choice for mapping of the biliary and vascular abnormalities in patients with PCC (Strength-1, Evidence-A).

13. MRC is as accurate as ERC in delineating biliary changes (Strength-1, Evidence-B).

14. MRC with MR portovenography demonstrates relationship of biliary changes with collaterals and the presence of shuntable vein. It also belps to distinguish between bile duct varices and common bile duct stones. (Strength1, Evidence-A)

15. EUS is useful when other imaging modalities are unrevealing or inconclusive in delineating the cause of biliary obstruction (Strength-2, Evidence-C).

16. EUS is helpful in delineating the type of choledochal collaterals. However, utility of EUS based delineation of choledochal collaterals needs to be defined in prospective studies (Strength-2, Evidence-C).

\section{NATURAL HISTORY AND PROGNOSIS}

\section{Natural History}

Data on natural history and prognosis of PCC are scarce. As discussed previously the course can be divided into asymptomatic and symptomatic phases. The majority (70-95\%) of patients in cross sectional studies have been asymptomatic, but long-term follow-up of these asymptomatic patients in longitudinal studies has not been reported. Only one study from Spain has reported longitudinal follow-up in 22 acute and 45 chronic noncirrhotic patients with PVT. ${ }^{29}$ Severity of cholangiographic changes was graded from grades I to III (Llop et $\mathrm{al}^{29}$; see 'Diagnosis' section above). Their observations suggested that PCC was a non-progressive, one-time event after acute
PVT. Overall, 73\% patients developed PCC at 33 months after acute PVT, which was similar to the frequency in the chronic PVT (80\%). Sixty percent of those imaged within 1 year of PVT had developed PCC; only one additional patients 1 among the 4 others developed PCC over 36 months. Changes did not progress in 3 of 4 patients followed for 43 months. Symptomatic PVT was seen only in patients with severe, grade III changes on cholangiography. However, in this study follow-up period was relatively short as the natural history of PCC may extend over 2 to 3 decades. Also, the severity of lesions even in grade III were much milder than that reported in Indian series which had evaluated patients after much longer duration since diagnosis of EHPVO. ${ }^{61}$

Patients with symptomatic PCC are normally older than patients presenting with EHPVO by median of 8-14 years, which suggests that PCC is a progressive condition and that long-term obstruction ${ }^{24,47,51,62}$ and prolonged portal hypertension are needed to cause changes severe enough to produce symptoms. Advanced biliary changes such as long strictures, and strictures with upstream stones have been reported in patients with EHPVO after prolonged durations of follow-up. ${ }^{47}$ Another study found that on sequential ERC studies performed a median of 29 months apart there was significant progression of biliary changes and $35 \%$ patients became symptomatic during this period. ${ }^{4}$ On prolonged follow-up patients with EHPVO have been reported to develop ascites, hypoalbuminemia and coagulopathy suggestive of liver dysfunction. ${ }^{11,63}$ This is postulated to be due to effects of chronic deprivation of portal blood flow to the liver leading to atrophy with a possible contribution from chronic cholestasis occurring due to PCC. Based upon these observations, the natural history of PCC can be divided into four stages (Table 4): 1. Preclinical: presence of portal cavernoma but no PCC with normal liver biochemistry and no symptoms of PCC; 2. Asymptomatic: early changes on cholangiography with normal or abnormal liver biochemistry but no symptoms; 3. Symptomatic: advanced changes on cholangiography with abnormal liver biochemistry and presence of symptoms without complications and; 4 . Complicated: Presence of liver dysfunction or fibrosis, extensive biliary changes like multifocal strictures, calculi above strictures, or biliopancreatic complications (Table 4). ${ }^{61}$

\section{Prognosis}

Patients with symptomatic PCC who achieve adequate stone clearance and biliary drainage endoscopically have been reported to have an excellent outcome in the available follow-up studies. ${ }^{20,51,64,65}$ The reported follow-up of surgically managed patients with PCC is relatively longer, ranging from 14 months to 12 years. Patients with symptomatic PCC who have had reversal of biliary changes and relief of symptoms also carry an excellent prognosis. ${ }^{46,66-68}$ 
Table 4 Stages in the Natural History of Portal Cavernoma Cholangiopathy.

\begin{tabular}{lcllcc}
\hline Stage & Portal cavernoma & Cholangiopathy & Liver biochemistry & Symptoms & Complications \\
\hline Preclinical & Present & Absent & Normal & Absent & Absent \\
Asymptomatic & Present & Early changes & Normal or abnormal & Absent & Absent \\
Symptomatic & Present & Advanced changes & Abnormal & Present & Absent \\
Complicated & Present & Advanced changes & Abnormal & Present & Present \\
\hline
\end{tabular}

However, patients without shuntable veins and advanced biliary changes of PCC cannot undergo shunt surgery. These patients may develop recurrent cholangitis ${ }^{20,48,51}$ and, eventually, liver dysfunction either due to secondary biliary cirrhosis or due to chronically diminished portal inflow. ${ }^{11,63}$ Attrition of hepatocellular function in these patients may reach the stage that they are candidates for liver transplantation. ${ }^{48,69-71} \mathrm{~A}$ detailed description of natural history and prognosis of PCC has been reviewed elsewhere in this issue. ${ }^{61}$

\section{Consensus Statement}

17. PCC develops early after acute PVT if recanalization is not achieved. (Strength-1, Evidence-B)

18. PCC is a progressive condition and symptoms may develop late in the course of portal bypertension. (Strength-1, Evidence-B)

19. PCC symptoms seem to occur only in patients with severe or advanced changes of cholangiopathy. (Strength-1, Evidence-B)

20. Presence of severe or advanced changes of cholangiopathy may be useful for identifying patients with a bigher risk of developing symptoms of PCC. (Strength-1, EvidenceB)

21. Patients with symptomatic PCC have good prognosis after successful endoscopic biliary drainage and after successful shunt surgery. (Strength-1, Evidence-A)

22. Patients with advanced biliary changes and without shuntable veins in whom repeated endoscopic biliary drainage are required carry a poor prognosis. These patients may have recurrent cholangitis and progressive liver dysfunction and might need liver transplantation. (Strength-1, Evidence-B)

\section{MANAGEMENT}

Asymptomatic patients with PCC do not require any treatment. Treatment of symptomatic PCC should be determined on a case by case basis. Primarily, it should be focused on the management of portal hypertension and relief of biliary obstruction.

Great care should be exercised in discriminating between symptomatic and asymptomatic patients. The label of symptomatic PCC should be applied only on clinical grounds, i.e. to patients with biliary pain, cholestatic jaundice and/or cholangitis due to either choledocholithiasis, choledochal stenosis or both. Patients with PCC and other associated abnormalities like deranged LFT but without the presentations mentioned above should be regarded as having asymptomatic PCC, and are not candidates for endoscopic therapy. Patients with symptomatic PCC, as described above, are candidates for the intervention, whether surgical or endoscopic. It is now accepted that the optimal management may require use of either or both these modalities as per the specific situation, though, as in other situations, surgical management is rarely resorted to as the first choice in patients with cholestatic jaundice and/or cholangitis. Initially endoscopic interventions in PCC were used to tackle emergency conditions like cholangitis due to strictures or stones using plastic stents or naso-bilairy drains to establish biliary drainage pending surgery. Subsequently endoscopic stone removal in PCC was demonstrated to be a safe procedure, with only few reports of hemobilia. ${ }^{47,48,51,64,65}$ Many patients are not candidates for surgery due to absence of a shuntable vein or liver dysfunction. These patients may require long-term endoscopic management in the form of repeated plastic stent exchanges ${ }^{47,51,65}$ or placement of removable covered metal stents. ${ }^{48,72}$ The role of peroralcholedochoscopy with Spyglass and laser or electrohydraulic lithotripsy for management of biliary calculi in complicated PCC needs to be explored.

Treatment of symptomatic PCC can be approached in a phased manner, coping first with biliary clearance; second, with portal decompression; and third, with persistent biliary obstruction. In patients with biliary obstruction with or without calculi, the first phase involves sphincterotomy and biliary drainage, with CBD stone removal if present. Sphincterotomy has not shown to be associated with increased bleed rates in PCC and use of Dormia baskets and balloon extractors has been shown to be safe. ${ }^{20,29,47,48,64}$ Hemobilia can be managed conservatively in most cases and is not more troublesome than in patients without PCC. ${ }^{48,51,73,74}$ Available literature suggests that endoscopic sphincterotomy with stone extraction is the first choice procedure for symptomatic CBD stones and endoscopic biliary drainage with plastic stents or nasobiliary tubes is the first choice in patients with cholangitis or cholestatic jaundice. $^{47,48,51,64,65}$ These are generally preoperative procedures, to be followed by porto-caval shunt surgery. Repeated stent exchanges over short periods of time (3-5 years) may occasionally result in resolution of stenosis but this option is usually resorted to as fall-back therapy 
for those with no surgical options for portal decompression. $^{47,51,65}$ Long-term results with this approach have not been reported. Anecdotal experience with the use of covered removable self-expandable metal stents is insufficient for any comment. Portal decompression surgery may be the first phase of therapy in patients in whom endoscopic intervention is not required such as a patient with isolated stenosis of CBD and with a shuntable vein. ${ }^{20} \mathrm{~A}$ more detailed discussion of the indications and results of endoscopy for initial drainage and stone removal as well as for long term management of PCC is reviewed elsewhere in this issue. ${ }^{75}$

The use of concomitant ursodeoxycholic acid (UDCA) has been reported to be beneficial by some authors. Perlemuter et $\mathrm{al}^{76}$ used UDCA in 5 of 8 patients with liver fibrosis or secondary biliary cirrhosis on liverbiopsy. Condat et $\mathrm{al}^{23}$ used UDCA in 3 of 4 patients with cholestasis who underwent endoscopic sphincterotomy and reported no recurrence of symptoms while on therapy. Llop et $\mathrm{al}^{29}$ used it in 10 of 14 patients with symptomatic PCC, including 5 patients with abdominal pain and cholestasis treated with UDCA alone, in two patients with stricture but no calculi and in 3 of 6 with choledochal stones after sphincterotomy and ductal clearance. They reported 'disappearance of symptoms and improve- ment in liver tests' in all treated patients during followup. However, other authors have reported equally good results with endoscopic management without UDCA and as no study comparing ERC alone versus ERC with UDCA in PCC is available, effect of UDCA in improving symptoms of PCC or altering its natural course is not clear.

In symptomatic PCC, the second phase involves portal decompression surgery by proximal splenorenal shunt or transjugular intrahepatic portosystemic shunt (TIPS). Shunt procedures result in regression of changes of cholangiopathy ${ }^{17,24}$ with complete regression of biliary obstruction occurring in about $62-88 \%$ of patients. ${ }^{17,46,47,66-68}$ Only $25-30 \%$ of patients will require further intervention for PCC after successful shunt procedure. Even if the regression of cholangiographic changes is incomplete, majority of the patients remain asymptomatic probably due to slowing-down of progression of PCC after shunt. Though the choice of shunt depends upon the surgeons' choice, non-selective shunts are preferred as they decompress the whole of the portal system allowing regression of collaterals in fact the majority of studies have reported results with proximal splenorenal shunts. ${ }^{46,66-68}$ A mesoRex bypass between the mesenteric vein and the left portal vein is preferred by some surgeons in children with EHPVO

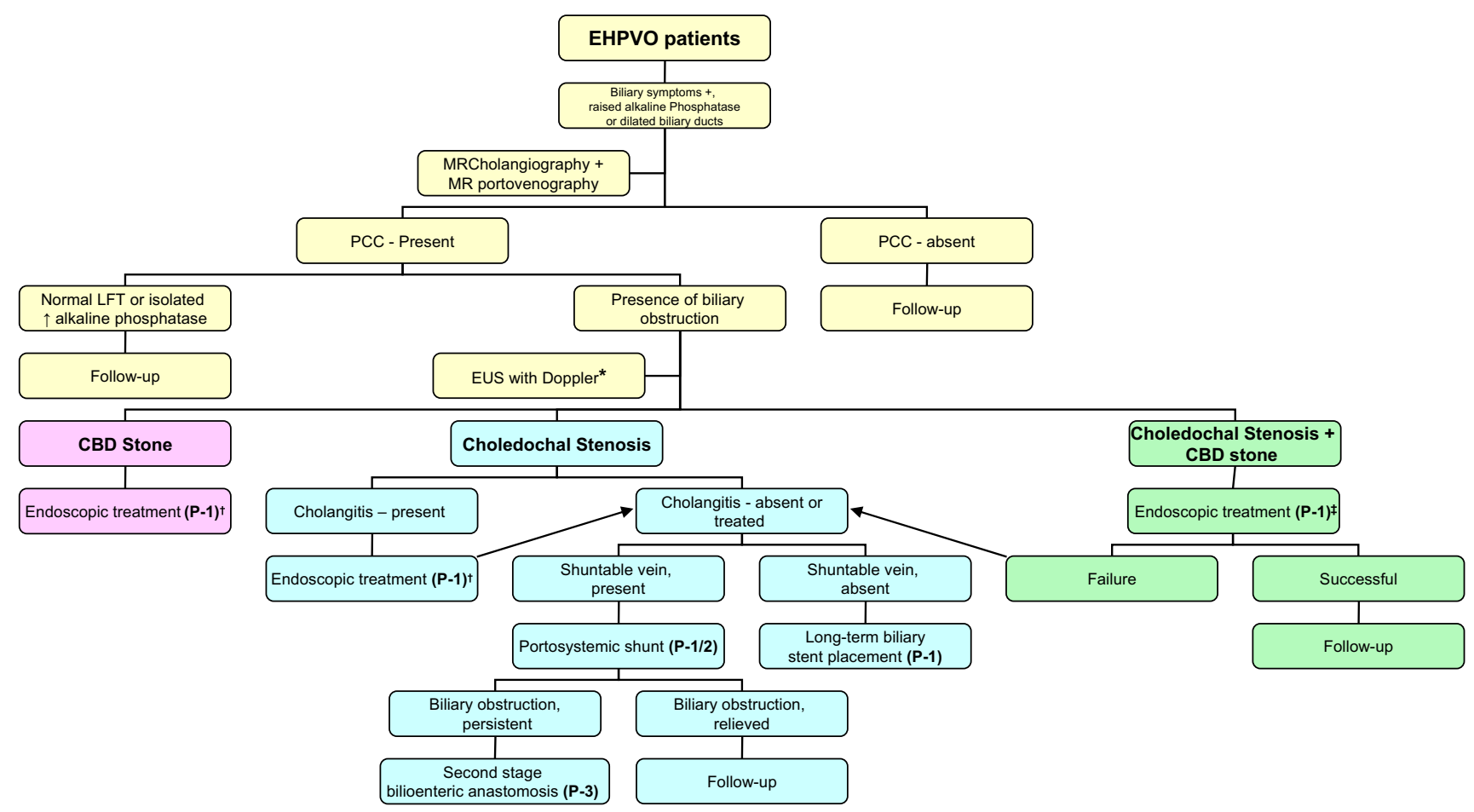

Figure 2 Algorithm for the management of a patient with portal cavernoma cholangiopathy. EHPVO, extrahepatic portal venous obstruction; CBD, common bile duct; LFT, liver function tests; MR, magnetic resonance; PCC, portal cavernoma cholangiopathy; *EUS with Doppler for evaluation of biliary obstruction when other imaging modalities are unrevealing; †endoscopic treatment includes endoscopic sphincterotomy, stone extraction with or without the help of mechanical lithotripsy, plastic biliary stent placement, etc; łendoscopic treatment includes endoscopic sphincterotomy and stricture dilatation, followed by stone extraction with or without the help of mechanical lithotripsy. P1, phase 1; P2, phase 2 and P3, phase 3. (Modified from Dhiman RK, et al. Gut 2007;56:1001-8). ${ }^{20}$ 
as it preserves portal blood flow to the liver and may improve growth in them. ${ }^{77-80}$

The third phase involves management of cases who continue to have biliary obstruction and remain symptomatic despite shunt procedure either due to blockage of shunt or due to persistent obstruction despite patent shunt. Second-stage biliary drainage surgery like hepaticojejunostomy or choledochoduodenostomy surgery on the bile ducts is performed in most of these cases with good resolution of symptoms as well as liver function tests. ${ }^{46,66-68}$ A more detailed discussion of the results of surgery in PCC is discussed in detail elsewhere in this issue. ${ }^{81}$ An alternative is approach is long-term endoscopic management of these patients with repeated stent exchanges and stone removal. ${ }^{46,66-68}$ No study has compared the result of these two approaches.

About $5-30 \%$ of patients with PCC did not have shuntable veins in several surgical series. ${ }^{46,47,66-68}$ Other patients may have advanced biliary changes of PCC that do not reverse fully with shunt surgery. These patients may develop recurrent cholangitis ${ }^{20,48,51}$ or liver dysfunction either due to secondary biliary cirrhosis or due to chronically diminished portal inflow. ${ }^{11,63}$ There have been case reports of such patients successfully undergoing liver transplantation. ${ }^{48,69-71,82}$ The portal inflow to the graft can be routed through the systemic circulation if there is extensive thrombosis of the splenoportal axis in the form of either a cavoportal hemi-transposition or a reno-portal inflow. This however does not decompress the splanchnic bed and symptoms related to hypersplenism and esophageal varices can persist despite transplantation.

An algorithmic approach for the management of PCC is presented in Figure 2.

\section{Consensus Statement}

23. Nasobiliary or biliary stent placement for acute cholangitis is safe and effective. (Strength-1, Evidence-A)

24. In the presence of normal platelet counts and coagulation, biliary sphincterotomy is a safe and effective procedure. (Strength-1, Evidence-A)

25. Biliary stricture dilatation and stent(s) placement is a safe procedure. (Strength-1, Evidence-B)

26. Biliary stricture dilatation and stone removal after lithotripsy may be performed safely. (Strength-2, Evidence-B)

27. Bilio-enteric anastomosis without prior surgery carries high morbidity and mortality and hence should not be performed. (Strength-1, Evidence-A)

28. Successful porto-systemic decompressive surgery results in reversal of symptoms and biliary strictures (partially or completely) in the majority of patients with PCC. Hence decompressive surgery should be performed if there is a shuntable vein available. (Strength-1, Evidence-A)
29. Non-selective shunts are preferred as they decompress the whole of the portal system allowing regression of collateral vessels. (Strength-1, Evidence-B)

30. Patients who continue to have biliary obstruction and remain symptomatic despite shunt procedure either due to blockage of shunt or due to persistent obstruction despite patent shunt. Second-stage biliary drainage surgery like hepaticojejunostomy or choledochoduodenostomy surgery on the bile ducts should be performed. (Strength-1, Evidence-B)

31. Secondary biliary cirrhosis is the only accepted indication for liver transplantation in patients with complicated PCC. Deceased donor liver transplantation is preferable over living donor liver transplantation as PCC is usually a non-emergency transplant and the graft can have portal blood inflow through a conduit to even a small segment of patent portal venous system or even to a cavernoma vessel. (Strength-2, Evidence-C)

\section{CONFLICTS OF INTEREST}

All authors have none to declare.

\section{REFERENCES}

1. Fraser J, Brown AK. A clinical syndrome associated with a rare anomaly of vena portal system. Surg Gynecol Obstet. 1944;78:520-524.

2. Malkan GH, Bhatia SJ, Bashir K, et al. Cholangiopathy associated with portal hypertension: diagnostic evaluation and clinical implications. Gastrointest Endosc. 1999;49:344-348.

3. Dhiman RK, Chawla Y, Vasishta RK, et al. Non-cirrhotic portal fibrosis (idiopathic portal hypertension): experience with 151 patients and a review of the literature. J Gastroenterol Hepatol. 2002;17:6-16.

4. Chandra R, Kapoor D, Tharakan A, et al. Portal biliopathy. J Gastroenterol Hepatol. 2001;16:1086-1092.

5. Chawla Y, Agrawal S. Portal cavernoma cholangiopathy - history, definition and nomenclature. J Clin Exp Hepatol. 2014;4: S15-S17.

6. Guyatt GH, Oxman AD, Vist GE, et al, GRADE Working Group. GRADE: an emerging consensus on rating quality of evidence and strength of recommendations. BMJ. 2008;336:924-926.

7. Hunt $\mathrm{AH}$. Compression of the common bile-duct by an enlarging collateral vein in a case of portal hypertension. $\mathrm{Br} J$ Surg. 1965;52:636-637.

8. Gibson JB, Johnston GW, Fulton TT, et al. Extrahepatic portal venous obstruction. Br J Surg. 1965;52:129-139.

9. Meredith HC, Vujic I, Schabel SI, O'Brien PH. Obstructive jaundice caused by cavernous transformation of the portal vein. $\mathrm{Br} J$ Radiol. 1978;51:1011-1012.

10. Hymes JL, Haicken BN, Schein CJ. Varices of the common bile duct as a surgical hazard. Am Surg. 1977;43:686-688.

11. Webb $\amalg$, Sherlock S. The aetiology, presentation and natural history of extra-hepatic portal venous obstruction. Q J Med. 1979;48:627-639.

12. Williams SM, Burnett DA, Mazer MJ. Radiographic demonstration of common bile duct varices. Gastrointest Radiol. 1982;7:69-70. 
13. Dan SJ, Train JS, Cohen BA, Mitty MA. Common bile duct varices: cholangio-graphic demonstration of a hazardous portosystemic communication. Am J Gastroenterol. 1983;78:42-43.

14. Spira A, Widrich WC, Keush KD, Jackson BT, Katzman HE, Coello AA. Bile duct varices. Arch Surg. 1985;1(20):1194-1196.

15. Choudhuri G, Tandon RK, Nundy S, Misra NK. Common bile duct obstruction by portal cavernoma. Dig Dis Sci. 1988;33:1626-1628.

16. Arotcarena R, Roulot D, Aubert A, Attali P, Benichou J, Coste T. Successful treatment by mesocaval anastomosis of a common-bileduct compression by choledochal varices. J Hepatol. 1995;23:629-630.

17. Dhiman RK, Puri P, Chawla Y, et al. Biliary changes in extrahepatic portal venous obstruction: compression by collaterals or ischemic? Gastrointest Endosc. 1999;50:646-652.

18. Lohr JM, Kuchenreuter S, Grebmeier H, Hahn EG, Fleig WE. Compression of the common bile duct due to portal vein thrombosis in polycythemia vera. Hepatology. 1993;17:586-592.

19. Sarin SK, Bhatia V, Makwana U. Poratal biliopathy in extrahepatic portal venous obstruction [abstract]. Indian J Gastroenterol. 1992;11(suppl 1):A82.

20. Dhiman RK, Behera A, Chawla YK, Dilawari JB, Suri S. Portal hypertensive biliopathy. Gut. 2007;56:1001-1008.

21. Dilawari JB, Chawla YK. Pseudosclerosing cholangitis in extrahepatic portal venous obstruction. Gut. 1992;33:272-276.

22. Bayraktar Y, Balkanci F, Kayhan B, et al. Bile duct varices or "pseudo-cholangiocarcinoma sign" in portal hypertension due to cavernous transformation of the portal vein. Am J Gastroenterol. 1992;87:1801-1806.

23. Condat B, Vilgrain V, Asselah T, et al. Portal cavernoma associated cholangiopathy: a clinical and MR cholangiography coupled with MR portography imaging study. Hepatology. 2003;37:1302-1308.

24. Dhiman RK, Chawla Y, Duseja A, et al. Portal hypertensive biliopathy (PHB) in patients with extrahepatic portal venous obstruction (EHPVO) [abstract]. J Gastroenterol Hepatol. 2006;21:A504.

25. Walser EM, Runyan BR, Heckman MG, et al. Extrahepatic portal biliopathy: proposed etiology on the basis of anatomic and clinical features. Radiology. 2011;258:146-153.

26. Rosenthal MD, White GH, Stephen MS, Gallagher JJ, Sandroussi C. Vascular biliopathy as a cause of common bile duct obstruction successfully treated by mesocaval shunt and endoscopic retrograde cholangiopancreatography biliary stent placement. Vascular. 2008;16:356-358.

27. Deltenre P, Valla DC. Ischemic cholangiopathy. Semin Liver Dis. 2008;28:235-246.

28. Bayraktar Y. Portal ductopathy: clinical importance and nomenclature. World J Gastroenterol. 2011;17:1410-1415.

29. Llop E, de Juan C, Seijo S, et al. Portal cholangiopathy: radiological classification and natural history. Gut. 2011;60:853-860.

30. Slieker JC, Farid WRR, van Eijck CHJ, et al. Significant contribution of the portal vein to blood flow through the common bile duct. Ann Surg. 2012;255:523-527.

31. Northover JM, Terblanche J. Bile duct blood supply. Its importance in human liver transplantation. Transplantation. 1978;26:67-69.

32. FurukawaH, Iwata R, Moriyama N, Kosuge T. Blood supply to the pancreatic head, bile duct and duodenum: evaluation by computed tomography during arteriography. Arch Surg. 1999;134:10861090.

33. Northover JM, Terblanche J. Applied surgical anatomy of the biliary tree. In: Blumgart LH, ed. The Biliary Tract. vol. 5. Edinburgh: Churchill Livingstone; 1982 [chapter 1].

34. Rameshbabu CS, Sharma M. Biliary tract anatomy and its relationship with venous drainage. J Clin Exp Hepatol. 2014;4:S18-S26.

35. Sharma M, Pathak A. Perforators of common bile duct wall with portal hypertensive biliopathy (with videos). Gastrointest Endosc. 2009;70:1041-1043.
36. De Gaetano AM, Lafortune M, Patriquin H, De Franco A, Aubin B, Paradis K. Cavernous transformation of the portal vein. Patterns of intrahepatic and splanchnic collateral circulation detected with Doppler sonography. AJR. 1995;165:1151-1155.

37. Kollmar O, Corsten M, Scheur C, Vollmar B, Schilling MK, Menger MD. Portal branch ligation induces a hepatic arterial buffer response, microvascular remodeling, normo-oxygenation, and cell proliferation in portal blood-deprived liver tissue. Am J Physiol Gastrointest Liver Physiol. 2007;292:G1534-G1542.

38. Nagino M, Nimura $\mathrm{Y}$, Kamiya J, Kanai M, Hayakawa $\mathrm{N}$, Yamamoto H. Immediate increase in arterial blood flow in embolized hepatic segments after portal vein embolization: CT demonstration. AJR. 1998;171:1037-1039.

39. Bayraktar Y, Balkanci F, Ozenc A, et al. The "pseudo-cholangiocarcinoma sign" in patients with cavernous transformation of the portal vein and its effect on the serum alkaline phosphatase and bilirubin levels. Am J Gastroenterol. 1995;90:20152019.

40. Dhiman RK, Singh P, Behera A, et al. Diagnosis of portal hypertensive biliopathy $(\mathrm{PHB})$ in patients with extrahepatic portal venous obstruction (EHPVO): endoscopic retrograde cholangiography versus MR cholangiography [abstract]. J Gastroenterol Hepatol. 2006;21:A507.

41. Sharma M, Pathak A. Intracholedochal varices in portal hypertensive biliopathy. Eur J Radiol. 2009;72:e119-e123.

42. Puri P. Pathogenesis of portal cavernoma cholangiopathy: is it compression by collaterals or ischemic injury to bile ducts during portal vein thrombosis? J Clin Hepatol. 2014;4:S27-S33.

43. Bechtelsheimer H, Conrad A. Morphology of cavernous transformation of the portal vein [author's translation] Leber Magen Darm. 1980;10:99-106.

44. Saji Y. The effect of decreased portal blood flow on the biliary system. Jpn J Surg. 1988;18:558-568.

45. Duseja A. Portal cavernoma cholangiopathy (PCC)—clinical characteristics. J Clin Exp Hepatol. 2014;4:S34-S36.

46. Agarwal AK, Sharma D, Singh S, Agarwal S, Girish SP. Portal biliopathy: a study of 39 surgically treated patients. HPB (Oxford). 2011:13:33-39.

47. Khare R, Sikora SS, Srikanth G, et al. Extrahepatic portal venous obstruction and obstructive jaundice: approach to management. J Gastroenterol Hepatol. 2005;20:56-61.

48. Oo YH, Olliff S, Haydon G, Thorburn D. Symptomatic portal biliopathy: a single centre experience from the UK. Eur J Gastroenterol Hepatol. 2009;21:206-213.

49. Khuroo MS, Yatoo GN, Zargar SA, et al. Biliary abnormalities associated with extrahepatic portal venous obstruction. Hepatology. 1993;17:807-813.

50. Nagi B, Kochhar R, Bhasin D, et al. Cholangiopathy in extrahepatic portal venous obstruction. Radiological appearances. Acta Radiol. 2000;41:612-615.

51. Sezgin O, Oguz D, Attintas E, et al. Endoscopic management of biliary obstruction caused by cavernous transformation of the portal vein. Gastrointest Endosc. 2003;68:602-608.

52. Chevallier P, Denys A, Novellas S, Schmidt S, Schnyder P, Bruneton JN. Magnetic resonance cholangiography features of biliary abnormalities due to cavernous transformation of the portal vein. Clin Imaging. 2006;30:190-194.

53. Bhatia V. Endoscopic retrograde cholangiography in Portal cavernoma cholangiopathy - results from different studies and Proposal for uniform terminology. J Clin Exp Hepatol. 2014;4:S37-S43.

54. Chawla Y, Dilawari JB, Katariya S. Gallbladder varices in portal vein thrombosis. AJR Am J Roentgenol. 1994;162:643-645.

55. Shin SM, Kim S, Lee JW, et al. Biliary abnormalities associated with portal biliopathy: evaluation on MR cholangiography. AJR Am J Roentgenol. 2007;188:W341-W347. 
56. Ozkavukcu E, Erden A, Erden I. Imaging features of portal biliopathy: frequency of involvement patterns with emphasis on MRCP. Eur J Radiol. 2009;71:129-134.

57. Kalra N, Shankar S, Khandelwal N. Imaging of portal cavernoma cholangiopathy. J Clin Exp Hepatol. 2014;4:S44-S52.

58. Palazzo L, Hochain P, Helmer C, et al. Biliary varices on endoscopic ultrasonography. Endoscopy. 2000;32:520-524.

59. Sharma M, Ponnusamy RP. Is balloon sweeping detrimental in portal biliopathy? A report of 3 cases. Gastrointest Endosc. 2009;70:171-173.

60. Sharma M, Rameshbabu CS. Portal cavernoma cholangiopathy: an endoscopic ultrasound based imaging approach. J Clin Exp Hepatol. 2014;4:S53-S61.

61. Kumar M, Saraswat VA. Natural history of Portal cavernoma cholangiopathy. J Clin Exp Hepatol. 2014;4:S62-S66.

62. Chandra R, Tharakan A, Kapoor D, Sarin SK. Comparative study of portal biliopathy in patients with portal hypertension due to different etiologies [abstract]. Indian J Gastroenterol. 1997;15(suppl 2):A59.

63. Rangari M, Gupta R, Jain M, et al. Hepatic dysfunction in patients with extrahepatic portal vein obstruction. Liver Int. 2003;23:434437.

64. Bhatia V, Jain AK, Sarin SK. Choledocholithiasis associated with portal biliopathy in patients with extrahepatic portal vein obstruction. Management with endoscopic sphincterotomy. Gastrointest Endosc. 1995;42:178-181.

65. Dumortier J, Vaillant E, Boillot O, et al. Diagnosis and treatment of biliary obstruction caused by portal cavernoma. Endoscopy. 2003;35:446-450.

66. Chattopadhyay S, Govindasamy M, Singla P, et al. Portal biliopathy in patients with non-cirrhotic portal hypertension: does the type of surgery affect outcome? HPB (Oxford). 2012;14:441-447.

67. Chaudhary A, Dhar P, Sarin SK, et al. Bile duct obstruction due to portal biliopathy in extrahepatic portal hypertension: surgical management. Br J Surg. 1998;85:326-329.

68. Vibert E, Azoulay D, Aloia T, et al. Therapeutic strategies in symptomatic portal biliopathy. Ann Surg. 2007;246:97-104.

69. Filipponi F, Urbani L, Catalano G, et al. Portal biliopathy treated by liver transplantation. Transplantation. 2004;77:326-327.
70. Hajdu CH, Murakami T, Diflo T, et al. Intrahepatic portal cavernoma as an indication for liver transplantation. Liver Transpl. 2007;13:1312-1316.

71. Gupta S, Singhal A, Goyal N, Vij V, Wadhawan M. Portal biliopathy treated with living donor liver transplant: index case. Exp Clin Transpl. 2011;9:145-149.

72. Layec S, D'Halluin PN, Pagenault M, Bretagne JF. Massive hemobilia during extraction of a covered self-expandable metal stent in a patient with portal hypertensive biliopathy. Gastrointest Endosc. 2009;70:555-556.

73. Tyagi P, Puri AS, Sharma BC. Balloon sweep in portal biliopathy [Letter] Gastrointest Endosc. 2010;71:885-886.

74. Sharma M, Babu CS, Dhiman RK, Chawla Y. Induced hypotension in the management of acute hemobilia during therapeutic ERCP in a patient with portal biliopathy. Gastrointest Endosc. 2010;72:1317-1319.

75. Saraswat VA, Rai P, Kumar T, Mohindra S, Dhiman RK. Endoscopic management of Portal cavernoma cholangiopathy: practice, principles and strategy. J Clin Exp Hepatol. 2014;4:S67-S76.

76. Perlemuter G, Bejanin H, Fritsch J, et al. Biliary obstruction caused by portal cavernoma: a study of 8 cases. J Hepatol. 1996;25:58-63.

77. Poddar U, Borkar V. Management of extra hepatic portal venous obstruction (EHPVO): current strategies. Trop Gastroenterol. 2011;32:94-102.

78. Lautz TB, Keys LA, Melvin JC, Ito J, Superina RA. Advantages of the meso-Rex bypass compared with portosystemic shunts in the management of extrahepatic portal vein obstruction in children. J Am Coll Surg. 2013;216:83-89.

79. Lautz TB, Sundaram SS, Whitington PF, Keys L, Superina RA. Growth impairment in children with extrahepatic portal vein obstruction is improved by mesenterico-left portal vein bypass. J Pediatr Surg. 2009;44:2067-2070.

80. Stringer MD. Improved body mass index after mesenterico-portal bypass. Pediatr Surg Int. 2007;23:539-543.

81. Varma V, Behera A, Kaman L, Chattopadhyay S, Nundy S. Surgical management of Portal cavernoma cholangiopathy. J Clin Exp Hepatol. 2014;4:S77-S84.

82. Gupta S, Taneja S. Liver transplantation for Portal cavernoma cholangiopathy. J Clin Exp Hepatol. 2014;4:S85-S87. 